\title{
Auftragsbearbeitung des Instituts für Qualität und Wirtschaftlichkeit im Gesundheitswesen (IQWiG)
}

Elske Jaskulla, Guido Noelle

Institut für Qualität und Wirtschaftlichkeit im Gesundheitswesen (IQWiG), Köln

psychoneuro 2005; 31 (6): 326-330

Nachdem das Institut für Qualität und Wirtschaftlichkeit im Gesundheitswesen (IQWiG) Ende 2004 seine Geschäftsräume in Köln bezogen hat und im Februar offiziell eröffnet wurde, erhielt es bereits im Dezember 2004 seine ersten Aufträge vom Gemeinsamen Bundesausschuss $(G-B A)$ nach Vorgaben des $S G B V \S 139 b$ Abs. 1, die das Institut gemäß der von ihm am 1. März 2005 veröffentlichten Methoden wissenschaftlich bearbeiten wird (http://www.iqwig.de/ media/_ber_uns/institut/files/2005_03_01_IQWiG_Methoden.pdf).

A ufgabe des Institutes ist es, themenbezogene Berichte zu erstellen sowie die Koordination und Publikation von wissenschaftlichen Untersuchungen in Bereichen, die einer Vervollständigung des versorgungsrelevanten medizinischen Wissens bedürfen, zu initiieren. Die ersten vom G-BA erhaltenen Aufträge sind im Einzelnen (Stand April 2005) in Tabelle 1 dargestellt.

\section{Zusammenarbeit mit} externen Sachverständigen

Mit den derzeit 35 Aufträgen ist das Institut in seiner jetzigen Aufbauphase mit knapp über 40 Mitarbeitern sicherlich für die nächste Zeit gut ausgelastet. Zur Erfüllung der gesetzlichen Aufgaben hat das Institut jedoch wissenschaftliche Forschungsaufträge verstärkt auch an externe Sachverständige zu vergeben. Dies kann Fragestellungen zur Literaturrecherche wie auch die Einbindung von Sachverständigen beim anschließenden Peer-Review betreffen. Aber auch schon bei der Detaillierung des Auftrags durch die
Definition der Zielkriterien kann externe Expertise erforderlich sein, um etwa im Einzelfall zu klären, was nun ein „patientenrelevanter Endpunkt" im Zusammenhang mit einer wissenschaftlichen Fragestellung ist.

Für die Mitarbeit als externer Sachverständiger ist zum einen der Nachweis der fachlich-medizinischen als auch methodischen Expertise bezüglich der jeweiligen wissenschaftlichen Fragestellung erforderlich. Die Darstellung der fachlichen und methodischen Expertise kann formlos durch die Darlegung praktischer Tätigkeiten, Lehre und Forschung und die Angabe wesentlicher Publikationen erfolgen. Diese Ausführungen werden ausschließlich den an den jeweiligen Projekten arbeitenden Personen offen gelegt.

Zum anderen müssen externe Sachverständige, die im Auftrag des Instituts tätig werden wollen, neben ihrer fachlichen und methodischen Expertise ihre fachlichen Abhängigkeiten gegenüber dem Institut darlegen. Sie haben dazu ihre jeweiligen Beziehungen zu Interessenverbänden, Auftragsinstituten, insbe- sondere der pharmazeutischen Industrie und der Medizinprodukteindustrie, einschließlich Art und Höhe von Zuwendungen offen zu legen. Als Interessenskonflikte werden dabei in Anlehnung an entsprechende Regelungen wissenschaftlicher medizinischer Zeitschriften insbesondere folgende Kriterien angesehen: Alle finanziellen Abmachungen, Beschäftigungen, Beratungen, Honorare, bezahlte ExpertenAussagen, Reise-Unterstützungen, Patent-Anträge sowie der Aktienbesitz innerhalb der letzten drei Jahre, die bzw. der die beauftragte Arbeit inhaltlich hätten beeinflussen können, sowie alle aktuellen persönlichen Beziehungen mit anderen Personen oder Organisationen, die die beauftragte Arbeit inhaltlich beeinflussen könnten (3). Die KriterienListe ist auch auf den Internetseiten des Instituts zu finden.

Das Institut hat zur Darlegung der fachlichen Abhängigkeiten kürzlich einen Fragebogen veröffentlicht (http://www.iqwig.de/de/auftrae ge/sachverstaendige/externe_Sach verstaendige.html). Die darin vorgesehene Aufführung potenzieller Interessenskonflikte dient vor allem der Transparenz und der damit verbundenen Versachlichung des wissenschaftlichen Prozesses bei der Erstellung der Berichte des Institutes und der Fachdiskussion darüber. Das Institut legt nur den am Berichtser- 
stellungsprozess beteiligten Personen einschließlich der Auftraggeber die potenziellen Interessenskonflikte anderer beteiligter Personen offen. Prinzipiell gibt es dabei keine Art der fachlichen Abhängigkeit, die ein absolutes Ausschlusskriterium für Sachverständige bedeutet. Das Institut bearbeitet allerdings nur Äußerungen von Sachverständigen inhaltlich, die eine vollständige Darlegung potenzieller Interessenskonflikte beinhalten. Alle Sachverständigen wie selbstverständlich die Mitarbeiter des Instituts auch -, die mittelbar oder unmittelbar im Auftrag des Institutes tätig werden und in den Erstellungsprozess der Berichte eingreifen, müssen daher potenzielle Interessenskonflikte vollständig und wahrheitsgemäß offen legen.

\section{Transparenz der Auftrags- bearbeitung}

Die Arbeit externer Sachverständiger muss auf der Grundlage der veröffentlichten Methoden erfolgen. Daraus geht z.B. hervor, dass bestimmte Arbeitsschritte, wie z.B. die Auswahl relevanter Publikationen im Rahmen der Informationsbeschaffung, mindestens durch zwei Personen durchgeführt werden müssen. Diese Aufgaben können daher nur von Arbeitsgruppen erledigt werden.

Im Rahmen der vom Institut veröffentlichten Methoden ist der Prozess der Auftragsbearbeitung stark formalisiert und transparent für Auftragnehmer und -geber dargestellt. Nur so kann künftig eine stringente und zügige Arbeit auf hohem qualitativem Niveau bei gleichzeitig begrenzten Ressourcen gewährleistet werden. Das Institut hat die Absicht, die durchschnittliche Dauer einer Auftragsbearbeitung auf deutlich unter ein Jahr zu straffen, was auch im internationalen Vergleich der HTA-Szene ein guter Wert wäre. Die einzelnen Schritte sind dabei in Abbildung 1 dargestellt.

Nach der Auftragsvergabe durch den G-BA oder das BMGS erfolgt die Bildung einer Projektgruppe unter Leitung des beauftragten Ressorts. Eine Person wird als verantwortliche Projektleiterin oder Projektleiter bestimmt. Die Zusammensetzung der Projektgruppe ist an dieser Stelle nicht fix, da sich durch die folgenden Arbeitsschritte Änderungsbedarf ergeben kann.

Sofern notwendig, erfolgt anschließend eine Konkretisierung des Auftrags in Abstimmung mit den zuständigen Gremien des Auftraggebers. Dies beinhaltet auch die erste (grobe) Definition der Zielkriterien, insbesondere der patientenrelevanten Endpunkte. Diese Definition wird im Weiteren durch die Projektgruppe verfeinert und es werden wissenschaftliche Fragestellungen formuliert. Hier ist im Einzelfall die Einbeziehung externer Expertise erforderlich, z.B. bei der Definition patientenrelevanter Endpunkte (2).

\section{Berichtsplan}

Anschließend erstellt die Projektgruppe den Berichtsplan. Der Berichtsplan beinhaltet, vergleich- bar mit dem Studienprotokoll einer geplanten klinischen Studie, die genaue wissenschaftliche Fragestellung einschließlich der Zielkriterien (z.B. patienten-relevante Endpunkte) sowie die Darlegung der Methodik der Beschaffung und Bewertung der Informationen (einschließlich der Ein- und Ausschlusskriterien der zu beschaffenden und zu bewertenden Informationen). Darüber hinaus werden Angaben über die sich anschließenden Schritte bei der Erstellung des Abschlussberichts, wie z.B. die Durchführung des Peer Review, Zeitpunkt der Veröffentlichung des Abschlussberichts etc. dargestellt, sofern diese zum Zeitpunkt der Erstellung des Berichtsplans bereits definiert wurden bzw. definiert werden können.

Die Erstellung des Berichtsplans ist gewöhnlich Aufgabe des Instituts,

\section{Abb. 1 Prozess der Auftragsbearbeitung}

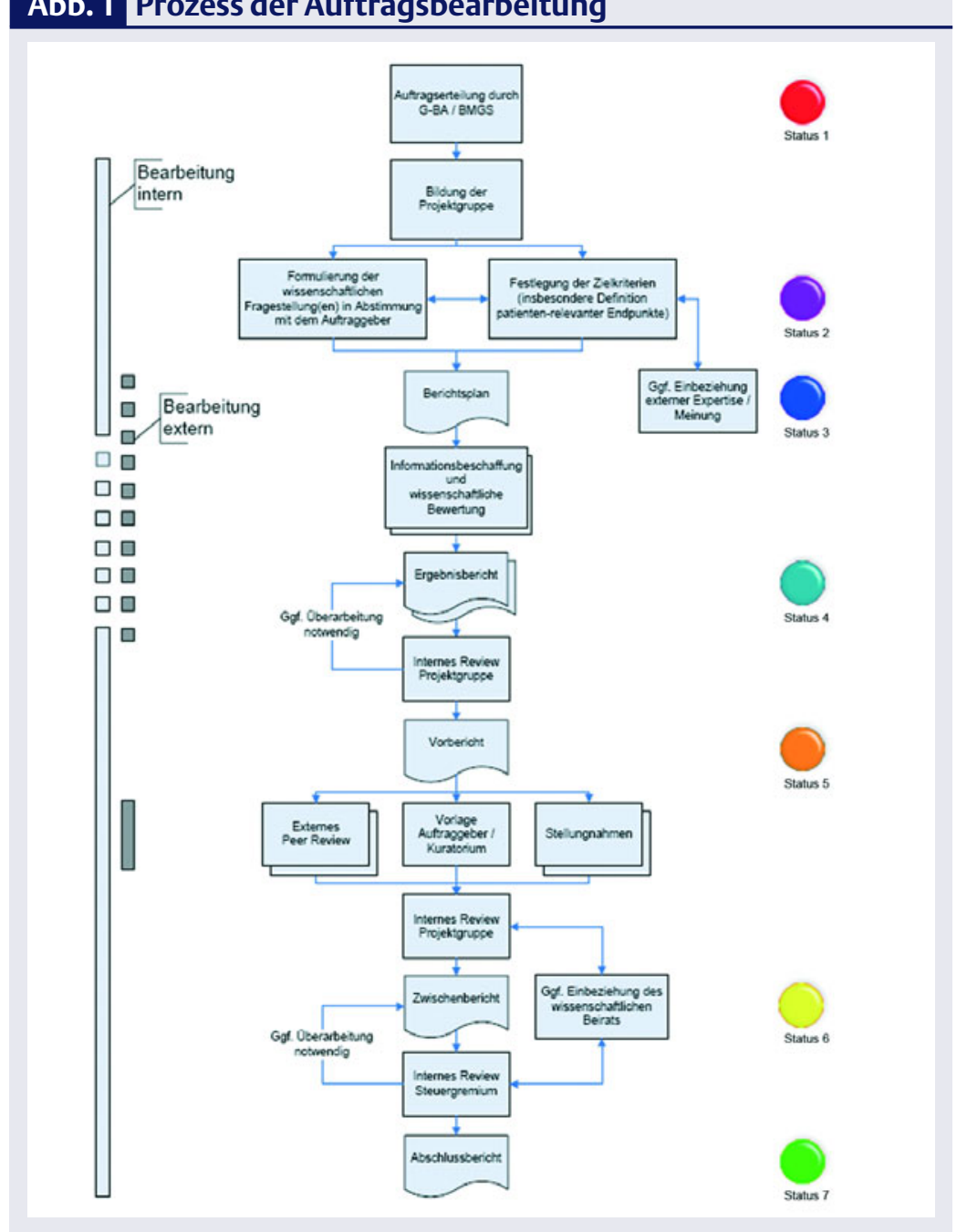




\section{Tab. 1 Aufträge des G-BA an das IQWiG, Stand April 2005}

\begin{tabular}{|c|c|}
\hline Nr. & Titel \\
\hline $\mathrm{A} 04 / 01$ & Nutzenbewertung von Clopidogrel bei Patienten mit kardialen und/oder vaskulären Erkrankungen \\
\hline $\mathrm{A} 04 / 02$ & Nutzenbewertung von L-Methionin bei Patienten mit neurogenen Blasenstörungen \\
\hline $\mathrm{A} 05 / 01$ & Nutzenbewertung lang wirksamer Insulinanaloga bei Patienten mit Typ1 Diabetes mellitus \\
\hline $\mathrm{A} 05 / 02$ & Nutzenbewertung kurz wirksamer Insulinanaloga bei Patienten mit Typ1 Diabetes mellitus \\
\hline A05/03 & Nutzenbewertung lang wirksamer Insulinanaloga bei Patienten mit Typ2 Diabetes mellitus \\
\hline A05/04 & Nutzenbewertung kurz wirksamer Insulinanaloga bei Patienten mit Typ2 Diabetes mellitus \\
\hline A05/05 & $\begin{array}{l}\text { Nutzenbewertung oraler Antidiabetika der Wirkstoffgruppen Alpha-Glukosidase-Hemmer, Biguanide, Glinide, Glitazone und } \\
\text { Sulfonylharnstoffe, einschließlich einer kombinierten Behandlung mit zwei oder mehr Medikamenten aus den genannten Wirk- } \\
\text { stoffgruppen sowie einer vergleichenden Bewertung untereinander }\end{array}$ \\
\hline $\mathrm{A} 05 / 06$ & Nutzenbewertung verschiedener nicht-medikamentöser Behandlungsstrategien bei Patienten mit Typ 2 Diabetes mellitus \\
\hline $\mathrm{A} 05 / 07$ & Nutzenbewertung einer langfristigen, normnahen Blutzuckersenkung bei Patienten mit Typ 2 Diabetes mellitus \\
\hline $\mathrm{A} 05 / 08$ & Nutzenbewertung der Urin- und Blutzuckerselbstmessung bei Patienten mit Typ 2 Diabetes mellitus \\
\hline $\mathrm{A} 05 / 09$ & $\begin{array}{l}\text { Vergleichende Nutzenbewertung von Diuretika, Beta-1-Rezeptor-selektiven Betablockern, ACE-Hemmern, Calcium-Antagonisten } \\
\text { und AT1-Antagonisten als Therapie der 1. Wahl bei Patienten mit Bluthochdruck }\end{array}$ \\
\hline $\mathrm{A} 05 / 10$ & $\begin{array}{l}\text { Nutzenbewertung einer langfristigen Blutdrucksenkung in den unteren normotonen Bereich bei Patienten mit Typ } 1 \text { Diabetes } \\
\text { mellitus und Patienten mit Typ } 2 \text { Diabetes mellitus }\end{array}$ \\
\hline $\mathrm{A} 05 / 11$ & Vergleichende Nutzenbewertung inhalativer Kortikosteroide zur Dauertherapie bei Patienten mit Asthma bronchiale \\
\hline $\mathrm{A} 05 / 12$ & $\begin{array}{l}\text { Nutzenbewertung lang wirksamer Beta-2-Rezeptor-Agonisten bei Patienten mit Asthma bronchiale, auch im Vergleich mit } \\
\text { anderen medikamentösen Therapieoptionen }\end{array}$ \\
\hline $\mathrm{A} 05 / 13$ & $\begin{array}{l}\text { Nutzenbewertung fixer Kombinationen aus Kortikosteroid und Beta-2-Rezeptor-Agonist zur inhalativen Anwendung bei Patienten } \\
\text { mit Asthma bronchiale }\end{array}$ \\
\hline $\mathrm{A} 05 / 14$ & $\begin{array}{l}\text { Nutzenbewertung von Leukotrien-Antagonisten bei Patienten mit Asthma bronchiale, auch im Vergleich mit anderen Therapie- } \\
\text { optionen }\end{array}$ \\
\hline A05/15 & $\begin{array}{l}\text { Nutzenbewertung inhalativer Kortikosteroide zur Dauertherapie bei Patienten mit chronisch obstruktiver Lungenerkrankung } \\
\text { (COPD) }\end{array}$ \\
\hline A05/16 & $\begin{array}{l}\text { Nutzenbewertung lang wirksamer Beta-2-Rezeptor-Agonisten bei Patienten mit chronisch obstruktiver Lungenerkrankung } \\
\text { (COPD), auch im Vergleich mit anderen medikamentösen Therapieoptionen }\end{array}$ \\
\hline A05/17 & $\begin{array}{l}\text { Nutzenbewertung fixer Kombinationen aus Kortikosteroid und Beta-2-Rezeptor-Agonist zur inhalativen Anwendung bei Patienten } \\
\text { mit COPD }\end{array}$ \\
\hline $\mathrm{A} 05 / 18$ & $\begin{array}{l}\text { Vergleichende Nutzenbewertung inhalativer Anticholinergika unterschiedlicher Wirkdauer bei Patienten mit chronisch obstruk- } \\
\text { tiver Lungenerkrankung (COPD) }\end{array}$ \\
\hline A05/19 & $\begin{array}{l}\text { Nutzenbewertung von Cholinesterase-Hemmern, ginkgohaltigen Präparaten und Memantin bei Patienten mit Demenz, auch im } \\
\text { Vergleich untereinander }\end{array}$ \\
\hline $\mathrm{A} 05 / 20$ & $\begin{array}{l}\text { Nutzenbewertung von trizyklischen Antidepressiva, Serotonin-Wiederaufnahme-Hemmern und Serotonin-Noradrenalin-Wieder- } \\
\text { aufnahme-Hemmern bei Patienten mit Depressionen, auch im Vergleich untereinander }\end{array}$ \\
\hline $\mathrm{A} 05 / 21$ & Nutzenbewertung verschiedener nicht-medikamentöser Behandlungsstrategien bei Patienten mit Bluthochdruck \\
\hline B05/01 & $\begin{array}{l}\text { Entwicklung eines Rechenmodells, wie unter Berücksichtigung deutscher Daten - zunächst für die Bereiche Kniegelenks- } \\
\text { endoprothetik und Koronarchirurgie im folgenden dann auch generell - Schwellenwerte für Mindestmengen festgelegt werden } \\
\text { können }\end{array}$ \\
\hline G05/01 & $\begin{array}{l}\text { Entwicklung eines Prognosemodells, Ermittlung der Auswirkung auf die Versorgung nach Schwellenwertermittlungen bei Min- } \\
\text { destmengen }\end{array}$ \\
\hline N04/01 & $\begin{array}{l}\text { Wissenschaftliche Bewertung des aktuellen medizinischen Wissenstands zu den verschiedenen nichtmedikamentösen, lokalen } \\
\text { Behandlungsmethoden der benignen Prostata-Hyperplasie }\end{array}$ \\
\hline N04/02 & $\begin{array}{l}\text { Wissenschaftliche Bewertung des aktuellen medizinischen Wissenstands zur interstitiellen Brachytherapie bei lokal begrenztem } \\
\text { Prostatakarzinom }\end{array}$ \\
\hline N04/03 & Wissenschaftliche Bewertung des aktuellen medizinischen Wissenstands zur Vakuum-Versiegelungstherapie von Wunden \\
\hline N04/04 & Wissenschaftliche Bewertung des aktuellen medizinischen Wissenstands zur Balneo-Photo-Therapie \\
\hline N05/01 & $\begin{array}{l}\text { Bei welchen Versorgungsformen führen Suprakonstruktionen im Vergleich zu nicht implantattragenden Versorgungsformen zu } \\
\text { einer Verbesserung der Kau-Funktion? }\end{array}$ \\
\hline N05/02 & $\begin{array}{l}\text { Wissenschaftliche Bewertung der Relevanz der Beschaffenheit der Gegenbezahnung bei der Versorgung mit festsitzendem } \\
\text { Zahnersatz }\end{array}$ \\
\hline N05/03 & Wissenschaftliche Bewertung des aktuellen medizinischen Wissenstands zur Stammzelltransplantation \\
\hline Q05/01 & $\begin{array}{l}\text { Erstellung von Evidenzberichten zum Verhältnis von Menge der erbrachten Leistungen und der Qualität bei PTCA und Bauch- } \\
\text { aortenaneurysma }\end{array}$ \\
\hline S05/01 & $\begin{array}{l}\text { Wissenschaftliche Bewertung des aktuellen medizinischen Wissenstands zur Früherkennungsuntersuchung von Sehstörungen } \\
\text { von Kindern bis zur Vollendung des } 6 \text {. Lebensjahres }\end{array}$ \\
\hline S05/02 & $\begin{array}{l}\text { Wissenschaftliche Bewertung des aktuellen medizinischen Wissenstands zur Früherkennungsuntersuchung von Hörstörungen } \\
\text { bei Neugeborenen }\end{array}$ \\
\hline
\end{tabular}


wobei dies unter Einbeziehung der externen Sachverständigen durchgeführt werden kann. Es ist in jedem Fall Aufgabe der externen Sachverständigen, den Berichtsplan sowohl aus ihrer fachlich-medizinischen als auch ihrer methodischen Sicht heraus zu prüfen und gegebenenfalls dem Institut Änderungs- oder Präzisierungsvorschläge zu unterbreiten. Erst nach diesem Arbeitsschritt ist eine Veröffentlichung des Berichtsplans über die Internetseiten des Instituts vorgesehen. Der gesamte Erstellungsprozess ist dabei einer internen Qualitätssicherung auf mehreren Ebenen unterworfen.

Begründete Änderungen am Berichtsplan erfolgen, wie auch bei einer klinischen Studie, möglichst in Form einer Nachbesserung (Amendment), die ebenfalls über die Internetseiten des Instituts veröffentlicht wird. Die im Berichtsplan getroffenen Angaben, insbesondere zu den Ein- und Ausschlusskriterien relevanter Informationen (z.B. wissenschaftlicher Literatur), sind von zentraler Bedeutung für die Kriterien, denen spätere Stellungnahmen genügen müssen. Grundsätzlich werden Stellungnahmen möglich sein, die sich beziehen auf

- die Vollständigkeit der Informationsbeschaffung und

- die Richtigkeit der wissenschaftlichen Bewertung dieser Informationen.

Der Berichtsplan ist Grundlage für die darauf folgende Informationsbeschaffung und wissenschaftliche Bewertung (2).

\section{Wissenschaftliche Bewertung}

Die Informationen, die einschließlich ihrer wissenschaftlicher Bewertung die Grundlage der Institutsberichte darstellen, können vielfältiger Art sein, z.B. wissenschaftliche Studien, Datensammlungen etc.

Die Informationsbeschaffung und wissenschaftliche Bewertung kann, zumindest in Teilen, durch einen oder mehrere externe Sachverständige ausgeführt werden. Die Aufgaben externer Sachverständiger bei der Durchführung einer Literaturrecherche können beim Institut angefordert

\section{Tab. 2 Erläuterung des Ampelsystems}

\begin{tabular}{|c|c|}
\hline 0000000 & $\begin{array}{l}\text { Auftragserteilung durch G-BA / BMGS } \\
\text { Konkretisierung der Fragestellung mit dem Auftraggeber }\end{array}$ \\
\hline 0000000 & Berichtsplan erstellt \\
\hline 0000000 & Ergebnisbericht erstellt \\
\hline 0000000 & Vorbericht erstellt \\
\hline 0000000 & Zwischenbericht erstellt \\
\hline 0000000 & Auftrag abgeschlossen / Abschlussbericht veröffentlicht \\
\hline
\end{tabular}

werden, die die Verfahrensweise im Einzelnen darlegen. Der Berichtsplan enthält eindeutige Ein- und Ausschlusskriterien, die aus den wissenschaftlichen Fragestellungen abgeleitetet werden. Sie beschreiben, welche Studien - bzw. allgemeiner, welche Informationen - zur Beantwortung der einzelnen Fragestellung jeweils potenziell relevant und daher von den externen Sachverständigen zur Bewertung heranzuziehen wären. Auf Grundlage dieser Ein- und Ausschlusskriterien ist von den externen Sachverständigen die Suchstrategie zu definieren und die Suche durchzuführen (1).

Die Bewertung der Studien ist nach den international üblichen wissenschaftlichen Methoden der Bewertung medizinischer Literatur durchzuführen (critical appraisal). Dabei ist für jede Publikation, die als relevant angesehen und daher einer Prüfung unterzogen wird, der vom Institut vorgegebene Extraktionsbogen in elektronischem Format auszufüllen. Dies beinhaltet eine präzise, kritische Würdigung jeder dieser Studien. Am Ende dieses Arbeitsschrittes steht der Ergebnisbericht, der neben einer Darlegung des medizinisch-fachlichen Hintergrunds die kritische Würdigung der einzelnen Studien an Hand der Extraktionsbögen sowie die vollständige Dokumentation der Literaturrecherche beinhaltet. Darüber hinaus sollten die externen Sachverständigen, neben dem Ergebnisbericht, dem Institut eine kurze, auf die im Berichtsplan genannten Zielkriterien fokussierte Einschätzung der Studienlage geben. Der Ergebnisbericht wird vom Institut mittels internen Peer Review auf Kongruenz mit dem Berichtsplan und den allgemeinen Methoden des Instituts geprüft (1).
Aus der Informationsbeschaffung und der sich daran anschließenden wissenschaftlichen Bewertung gehen je nach Zahl der externen Sachverständigen ein oder mehrere $\mathrm{Er}$ gebnisberichte hervor, die nach internem Review durch die Projektgruppe zum Vorbericht zusammengefasst werden. Dieser Vorbericht wird dann in der Regel zeitgleich

- einem oder mehreren externen Peer Reviewern mit ausgewiesener methodischer und/oder fachlicher Kompetenz zugeleitet - dem Auftraggeber und Kuratorium, auch zur Frage der Vollständigkeit in Bezug auf den ursprünglich erteilten Auftrag, zugeleitet

- auf den Internetseiten des Instituts veröffentlicht, um Stellungnahmen, die den dort genannten Kriterien genügen müssen, einzuholen.

Je nach Art und Umfang der eingegangenen Stellungnahmen einschließlich der Ergebnisse des Peer Reviews sowie der Stellungnahmen des Auftraggebers und des Kuratoriums kann anschließend optional eine Diskussion unter Teilnahme der externen Sachverständigen durchgeführt werden, sofern dies der Verbesserung der wissenschaftlichen Qualität des späteren Abschlussberichts dienlich erscheint. Daran anschlieBend erstellt die Projektgruppe einen Zwischenbericht, der dem Steuergremium - bestehend aus der Institutsleitung und den Ressortleitern des Instituts - zum internen Review vorgelegt wird. Der sich daran anschließende Abschlussbericht wird dann dem Auftraggeber zugeleitet und nach dessen Zustimmung, spätestens aber nach einer im Berichtsplan vorab festgelegten Latenzzeit, durch das Institut veröffentlicht (2). 
Das Institut hat in seinen Methoden festgelegt, alle Ergebnisse, die innerhalb des Instituts oder im Auftrag des Instituts erarbeitet wurden, transparent zu machen und zu veröffentlichen. Zur Wahrung der Unabhängigkeit des Institutes muss ausgeschlossen werden, dass die Auftraggeber oder andere interessierte Dritte Einfluss auf die Inhalte der Berichte nehmen können. Dies könnte zu einer Vermengung der wissenschaftlichen Ergebnisse einerseits und politischen oder interessengesteuerten Aspekten andererseits führen. Gleichzeitig muss vermieden werden, dass das Institut seinerseits bestimmte Ergebnisse zurückhält. Um dies auszuschließen, werden alle Ergebnisse, die das Institut im Rahmen seiner gesetzlichen Aufgaben erarbeitet, einschließlich des Berichtsplans und einer Zusammenfassung der zugrunde liegenden Materialien, die zur Ergebnisfindung beigetragen haben, zeitnah veröffentlicht.

Die Rechte an der Publikation liegen, falls nicht anders vereinbart, beim Institut (2).

Das IQWiG hat im Januar diesen Jahres mit der Bearbeitung der ersten Aufträge des G-BA begonnen. Auf den Internetseiten des IQWiG kann man erfahren, welche Aufträge momentan bearbeitet werden und in welchem Bearbeitungsstatus sich der jeweilige Auftrag befindet. Dazu wurde ein Ampelsystem entwickelt, welches jedem Bearbeitungsschritt einen bestimmten Status zuordnet. Der gesamte Prozess wird dabei in sieben wesentliche Phasen unterteilt.

Interessierte Personen oder Institutionen können ihr Angebot zur Mitarbeit an einzelnen Projekten an die Institutsleitung richten. Die erforderlichen Unterlagen dazu sind auf den Internetseiten des Instituts zu finden (http://www.iqwig.de/de/ auftraege/sachverstaendige/externe_Sachverstaendige.html).

Das Steuergremium des Instituts wählt dann anhand der eingegangenen Unterlagen geeignete Kooperationspartner gemäß den beschriebenen Kriterien aus. Die Vergütung externer Sachverständiger erfolgt dabei nach vom Vorstand festgelegten Tagessätzen in einem zuvor vereinbarten Mengengerüst.

Das IQWiG hat einen Mustervertrag für die Mitarbeit externer Sachverständiger erarbeitet, der bei Interesse beim Institut angefordert werden kann bzw. in Kürze ebenfalls auf den Internetseiten des Instituts abgerufen werden kann. In diesem Vertragsentwurf ist alles Weitere zu den Inhalten der Leistungserbringung, der Vergütung und zu Publikationsrechten geregelt. Der Berichtsplan ist dabei immer ein wesentlicher und fester Vertragsbestandteil.

\section{Literatur}

1. Biester K, Follmann M, Kaiser T, Kirchner H, Lange P, Peinemann F, Stich AK. Aufgaben externer Sachverständiger - Literaturrecherche 29.03.2005

2. Bastian H, Bender R, Kaiser T, Kirchner H, Lange S, Müller de Cornejo G, Noelle G, Sawicki P. Methoden Version 1.01.03.2005

3. James A, Horton R. The Lancet's policy on conflicts of interest. Lancet 2003; 361:8-9

\section{Korrespondenzadresse:}

Institut für Qualität und Wirtschaftlichkeit im Gesundheitswesen (IQWiG)

Dr. med. Guido Noelle

Dillenburger Straße 27

51105 Köln

guido.noelle@iqwig.de
Georg Thieme Verlag KG

Rüdigerstraße 14

70469 Stuttgart

Telefon (0711) 8931-0

Telefax (0711) 8931-322

Verantwortliche Schriftleitung:

Markus Gastpar, Essen

jürgen Fritze, Pulheim

Erweiterte Schriftleitung:

Günther Faust, Mainz

Manfred Fichter, Prien am Chiemsee

Andreas Hufnagel, Essen

Joachim Morgner, Dresden

\section{Wissenschaftlicher Beirat:}

Thomas Becker, Günzburg; Stefan Bender, Marsberg; Horst Berzewski, Berlin; Lotha Blaha, Deggendorf; Hans-Dieter Brenner Bern; Pasquale Calabrese, Bochum; Klemens Dieckhöfer, Bonn; Tilman Elliger, Köln; Volker Faust, Ravensburg; Franz joseph Freisleder, München; Michael Geyer, Leipzig; Ralf Gold, Göttingen; Iver Hand, Hamburg; Kurt Heinrich, Düsseldorf; Andreas Heinz, Berlin; Paul Hoff, Zürich; Wolfgang Jost, Wiesbaden; Georg Juckel, Berlin; Siegfried Kaumeier, Merzig; Wilfried Kuhn, Schweinfurt; Gerd Laux, Wasserburg/München; Matthias R. Lemke, Bonn; Walter E. Müller, Frankfurt am Main; ThoWalter E. Müller, Frankfurt am Main;
mas Müller, Bochum; Dieter Naber, mas Müller, Bochum; Dieter Naber,
Hamburg; Hartmut Radebold, Kassel; Helmut Remschmidt, Marburg; Peter Rie derer, Würzburg; Eckart Rüther, Göttingen; Jörn Peter Sieb, Stralsund; Wolfgang Weig, Osnabrück; Wolfgang Werner, Merzig; Roland Wörz, Bad Schönborn; Manfred Wolfersdorf, Bayreuth

Bestellungen: Über den Buchhandel oder direkt beim Verlag

Bezugsbedingungen:

Einzelheft $€ 10,50$ + Porto

Jahresabonnement $€ 75$,-

inkl. MwSt. und Porto.

Für Medizinstudenten

gegen Nachweis und Bankeinzug

€ 48,- inkl. MwSt. und Porto.

Ausland zuzüglich Versandkosten

(cash with order) $€ 19,80$ (Europa)

bzw. $€ 52,80$ (Airlift).

Der Abonnementpreis umfasst $12 \mathrm{Ka}$ lendermonate (Mindestlaufzeit). Abonnements laufen weiter, wenn nicht 3 Monate vor Jahresende eine Abbestellung beim Verlag vorliegt.

Bei Nichtbelieferung im Falle höherer Gewalt, bei Störungen des Betriebsfriedens, Arbeitskampf (Streik, Aussperrung) bestehen keine Ansprüche gegen rung) bestag.

\section{Verlag und Copyright:}

(c) 2005 by Georg Thieme Verlag,

Rüdigerstraße 14, 70469 Stuttgart.

Mit dem Abdruck des Beitrages erwirbt der Verlag das alleinige und ausschließliche Recht für die Veröffentlichung in sämtlichen Publikumsmedien sowie Übersetzungen in fremde Sprachen. Nachdruck, fotomechanische Wiedergabe und Speicherung in den Datenverarbeitungsanlagen, auch auszugsweise, nur nach schriftlicher Genehmigung des Verlages. Die Abbildungen in den Beiträgen lages. Die Abbildungen in den Beiträgen
stammen, wenn nicht anders vermerkt, stammen, wenn nicht anders vermerkt,
von den jeweiligen Autoren. Für Inhalt, von den jeweiligen Autoren. Für Inhalt,
Vorschau und Titelseite werden AbbilVorschau und Titelseite werden Abbil-
dungen aus den beschriebenen Arbeiten dungen aus den beschriebenen Arbeiten
verwendet, anderenfalls ist die Herkunft ebenfalls im Inhaltsverzeichnis deklariert. Hinweis für Autoren:

Zur Veröffentlichung in der Zeitschrift anZur Veroffentlichung in der Zeitschrift anOriginalarbeiten, Übersichtsarbeiten und Originalarbeiten, Ubersichtsarbeiten und
Kasuistiken, die nicht auch gleichzeitig an anderer Stelle zur Veröffentlichung eingereicht sein dürfen. Richtlinien zur Abfassung des Manuskriptes können bei der Redaktion angefordert werden.

Die eingereichten Arbeiten für Editorial, Schwerpunkt/Brennpunkt und Übersicht/Originalarbeit werden einem peer review der Schriftleitung unterzogen. Supplements liegt in der Verantwortung der Redaktion.

\section{Redaktionsleitung: \\ Günther Buck \\ Telefon (0711) 89 31-440 \\ Chefredaktion: \\ Katrin Wol \\ Telefon (0711) 89 31-179 \\ Katrin.Wolf@thieme.de \\ Redaktionsassistenz: \\ Sabine Bischoff \\ Telefon (0711) 8931-551 \\ Telefax (0711) 89 31-322}

Internet-Adresse:

http://www.psychoneuro.info

Verantwortlich für den Anzeigenteil:

pharmedia, Anzeigen-

und Verlagsservice $\mathrm{GmbH}$

Anzeigenleitung:

Greta Weller

Telefon (0711) 89 31-304

Greta.Weller@pharmedia.de

Zur Zeit gilt Anzeigenpreisliste

1.10.2004

Zeitschriftenvertrieb:

Telefon (0711) 89 31-321

Herstellung:

Werner Schulz

Telefon (0711) 89 31-331

Satz, Grafik, Layout:

Wolfgang Eckl, Werner Schulz,

Karl-Heinz Zobel

Druck und Verarbeitung:

W. Kohlhammer Druckerei GmbH + Co. 70329 Stuttgart

Bankverbindung:

Deutsche Bank Stuttgart

Konto-Nr. 1420 017, BLZ 60070070

Landesbank Baden-Württemberg

Konto-Nr. 2055 723, BLZ 60050101

Postgiro Stuttgart

Konto-Nr. 45 000-705, BLZ 60010070

Erscheinungsweise: monatlich

Regularly listed in EMBASE

\section{Hinweis:}

Wie jede Wissenschaft ist die Medizin ständigen Entwicklungen unterworfen. Forschung und klinische Erfahrung erweitern unsere Erkenntnis, insbesondere was Behandlung, medikamentöse Therapie sowie Diagnostik (Laborwerte etc.) anbelangt. Soweit in dieser Zeitschrift Dosierungen, Applikationen oder Laborwerte erwähnt werden, darf der Leser zwar darauf vertrauen, dass Autoren, Herausgeber und Verlag große Sorgfalt darauf verwandt haben, dass diese An.

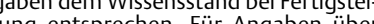
Do Dosierungsanweisungen, Applikationsorm Verlag jedoch keine Gewähr übernommen werden. Jeder Benutzer ist angehalten, durch sorgfältige Prüfung der Beipackzettel der verwendeten Präparate und ggf. nach Konsultation eines Spezialisten festzustellen, ob die dort gegebene Empfehlung für Dosierungen oder die Beachtung von Kontraindikationen Beachen

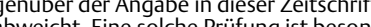
abweicht. Eine solche Prüfung ist besonders wichtig bei selten verwendeten Prăparaten oder solchen, die neu au den Markt gebracht worden sind. Jede Dosierung oder Applikation erfolgt auf eigene Gefahr des Benutzers. Laborwerte müssen immer auf Ihre Plausibilität geprüft werden und sind abhängig vom jeweiligen Testgerät bzw. Testkit. Autoren und Verlag appellieren an jeden Benutzer, ihm auffallende Ungenauigkeiten dem Verlag mitzuteilen. 